\title{
The phosphodiesterase- 4 and glycine transporter-1 inhibitors enhance in vivo hippocampal theta network connectivity and synaptic plasticity, whereas D-serine does not
}

\author{
A. Ahnaou (1) T. Broadbelt ${ }^{1}$, R. Biermans ${ }^{1}$, H. Huysmans', N. V. Manyakov $\mathbb{E}^{1}$ and W. H. I. M. Drinkenburg
}

\begin{abstract}
Dysfunctional N-methyl-D-aspartate receptors (NMDARs) and cyclic adenosine monophosphate (CAMP) have been associated with deficits in synaptic plasticity and cognition found in neurodegenerative and neuropsychiatric disorders such as Alzheimer's disease (AD) and schizophrenia. Therapeutic approaches that indirectly enhance NMDAR function through increases in glycine and/or D-serine levels as well as inhibition of phosphodiesterases that reduces degradation of CAMP, are expected to enhance synaptic strength, connectivity and to potentially impact cognition processes. The present in vivo study investigated effects of subcutaneous administration of D-serine, the glycine transporter 1 (GlyT1) inhibitor SSR504734 and the PDE4 inhibitor rolipram, on network oscillations, connectivity and long-term potentiation (LTP) at the hippocampi circuits in Sprague-Dawley rats. In conscious animals, multichannel EEG recordings assessed network oscillations and connectivity at frontal and hippocampal CA1-CA3 circuits. Under urethane anaesthesia, field excitatory postsynaptic potentials (fEPSPS) were measured in the CA1 subfield of the hippocampus after high-frequency stimulation (HFS) of the Schaffer collateral-CA1 (SC) pathway. SSR504734 and rolipram significantly increased slow theta oscillations $(4-6.5 \mathrm{~Hz})$ at the $\mathrm{CA} 1-\mathrm{CA}$, slow gamma oscillations $(30-50 \mathrm{~Hz})$ in the frontal areas and enhanced coherence in the CA1-CA3 network, which were dissociated from motor behaviour. SSR504734 enhanced short-term potentiation (STP) and fEPSP responses were extended into LTP response, whereas the potentiation of EPSP slope was short-lived to STP with rolipram. Unlike glycine, increased levels of D-serine had no effect on network oscillations and limits the LTP induction and expression. The present data support a facilitating role of glycine and CAMP on network oscillations and synaptic efficacy at the CA3-CA1 circuit in rats, whereas raising endogenous D-serine levels had no such beneficial effects.
\end{abstract}

\section{Introduction}

$\mathrm{N}$-methyl-D-aspartate receptors (NMDARs) and cyclic adenosine monophosphate (cAMP) play a pivotal role in plastic mechanisms of learning and memory ${ }^{1}$. Dysfunctional NMDARs and cAMP signalling have been associated with deficits in synaptic plasticity and cognitive decline found in neuropsychiatric and neurodegenerative disorders such as schizophrenia and Alzheimer's disease $(\mathrm{AD})^{2-4}$.

\footnotetext{
Correspondence: A. Ahnaou (aahnaou@its.jnj.com)

${ }^{1}$ Department of Neuroscience, Janssen Research \& Development, A Division of Janssen Pharmaceutica NV, Turnhoutseweg 30, B-2340 Beerse, Belgium
}

Therapeutic approaches that enhance NMDAR function through increases in endogenous ligands of the NMDAR, as well as inhibition of phosphodiesterases, which reduces degradation of cAMP, are expected to enhance endogenous neurorepair and synaptic strength to potentially impact cognition processes $^{5-7}$.

The strength of the glutamatergic neurotransmission is tightly controlled by the synaptic concentration of glycine and D-serine near NMDA receptors. Glycine and Dserine are endogenous ligands at the glycine $B$ site of the NMDA receptor, which act as a requisite co-agonist of 
glutamate for the activation of this receptor ${ }^{8}$. Glycine, which generally acts as an inhibitory neurotransmitter, has an excitatory activity at the strychnine-insensitive coagonist site $^{8}$. D-serine, which is released from astrocytes is more potent at the strychnine-insensitive binding site than glycine9. On the one hand, levels of synaptic glycine are tightly controlled by the specific transporter GlyT1 localized on glial cells and neurons closely associated with the NMDA receptor ${ }^{10}$. Several well tolerated, high affinity GlyT1 inhibitors have been developed and shown to increase central glycine levels for a positive functional impact on central glutamatergic transmission and to possess the preclinical profile of putative antipsychotics properties in preclinical animal models ${ }^{11-14}$. On the other hand, reducing D-serine levels impairs NMDAR-mediated processes in several structures, including the hippocampus, prefrontal cortex, nucleus accumbens or amygdala. Functional studies using enzymatically, or genetically induced depletion of D-serine showed reduction of synaptic NMDARs currents and thereby alteration in synaptic plasticity at the level of the hippocampus ${ }^{9,15,16}$, amygdala ${ }^{17}$, and nucleus accumbens $^{18}$, the retina ${ }^{19}$ and the hypothalamus ${ }^{20}$. The role of D-serine at NMDARs is further illustrated by studies showing that synaptic and cognitive impairments during aging is linked to a downregulation of D-serine synth$\mathrm{esis}^{21,22}$. Detailed analysis of the contribution of the two co-agonists in the regulation of NMDARs at the hippocampus CA1 level revealed that D-serine would preferentially act on synaptic NMDARs whilst glycine would modulate extra-synaptic NMDARs ${ }^{15}$.

The integrity of the hippocampal formation is critical for normal memory function, hence much experimental interest focused to characterize structural and functional changes of the hippocampus throughout aging and in disease animal models. Key mechanisms proposed to explain impaired cognitive processing are associated with deficits of network oscillations at the fronto-hippocampal circuit and impaired synaptic plasticity related to longterm potentiation (LTP) ${ }^{23,24}$.

Network oscillations represent fundamental mechanisms enabling coordinated activity between multiple association regions during normal brain functioning. Hippocampal theta oscillations have been found to drive processing in the prefrontal cortex ${ }^{24,25}$. Increased gamma band $(30-100 \mathrm{~Hz})$ oscillations occur during the transient brain states that are associated with attention and stimulus recognition ${ }^{26,27}$. More recently, several studies have suggested that gamma oscillations nested within theta $(4-12 \mathrm{~Hz})$ oscillations play a role in working memory functions ${ }^{24}$. Also, substantial data suggest that corticothalamic $^{28,29}$ and hippocampal networks ${ }^{30}$ make use of beta $(12-30 \mathrm{~Hz})$ and gamma $(30-100 \mathrm{~Hz})$ frequency band activities for long-distance transmission of information among task-related brain sites, although a number of those studies were carried out in brain slices or animal model of diseases ${ }^{31-33}$.

LTP is most commonly induced by a combined activation of $\alpha$-amino-3-hydroxy-5-methyl-4-isoxazolepropionic acid (AMPAR) and NMDA receptors. NMDAR-activitydependent LTP is suggested as a mechanism for short- and long-term memory acquisition ${ }^{34,35}$. Presynaptic depolarisation leads to exocytosis of glutamate into the synaptic cleft, activates many of the postsynaptic proteins, including the cAMP. CAMP/PKA and cyclic guanosine monophosphate (cGMP)/protein kinase G (PKG) pathways involved in the LTP expression, maintenance and memory enhancement ${ }^{36}$. To regulate the signalling of both pathways, the phosphodiesterase (PDE) enzyme family hydrolyses cAMP and cGMP preventing kinase activity ${ }^{3}$. There are $11 \mathrm{PDE}$ subgroups found in varying levels across the nervous system. There is a strong case for the regulation of synaptic plasticity by PDE4, which is the most widely studied PDE and is selective for cAMP over cGMP. PDE4 hydrolyses cAMP and is found in the hippocampus and cortex among other areas in rodents ${ }^{37}$. Rolipram exhibits memory-enhancing effects in rodents. A decrease of PDE4 isoforms has been shown in AD patients and the PDE4 inhibitor rolipram has demonstrated memory enhancements ${ }^{36}$ as well as displaying a good antidepressant effect but with unpleasant side effects ${ }^{38}$. PDE4 inhibition rescues impaired LTP and prevents object recognition memory deficits in an animal model of psychosis ${ }^{39}$.

In the present work, we modulated D-serine, glycine and cAMP levels to ascertain their functions in network oscillations and synaptic plasticity in healthy rats. By increasing synaptic concentration of glycine or D-serine in the vicinity of NMDA receptors, blockade of GlyT1 and/or D-serine are expected to potentiate glutamatergic transmission. We demonstrate that increased levels of glycine and cAMP increased hippocampal network activity and LTP in healthy rats. Under physiological conditions, synaptic plasticity in vivo at CA1 did not require high levels of $\mathrm{D}$-Serine.

\section{Materials and methods \\ Animals}

All experimental procedures were conducted in strict accordance with the guidelines of the Association for Assessment and Accreditation of Laboratory Animal Care International (AAALAC), and with the European Communities Council Directive 2010/63/UE of 22/09/2010 and were approved by local ethical committee. Experiments were carried out on Sprague-Dawley male rats 180-250 g (Harlan Nederland) housed in individually ventilated cages located in a sound-attenuated chamber under the controlled light/dark cycle (light: 7.00-19.00) with standard food and water available ad libitum. 


\section{Surgery and electrode implantation Network oscillations and connectivity}

Surgery was performed under Isoflurane anaesthesia as described earlier ${ }^{40,41}$. In brief, animals were equipped with two stainless steel fixing screws (diameter $1 \mathrm{~mm}$ ) for the recording of frontal electroencephalographic activities (EEG) inserted bilaterally in the left and right cortex (FL/ FR: AP $+2 \mathrm{~mm}, \mathrm{~L} \pm 2 \mathrm{~mm}$ from Bregma). Four stainless steel wires used for intra-hippocampal electrodes (CA1: AP $-3.14 \mathrm{~mm}, \quad \mathrm{~L} \pm 1.8 \mathrm{~mm}, \mathrm{~V}+2.8 \mathrm{~mm}$; CA3: AP $-4.5 \mathrm{~mm}, \mathrm{~L} \pm 3.8 \mathrm{~mm}, \mathrm{~V}+4 \mathrm{~mm}$ from Bregma, respectively). In addition, stainless steel wires (7N51465T5TLT, $51 / 46$ Teflon Bilaney, Germany) were placed in the muscle of the neck to record the electromyogram activity (EMG). Electrodes were connected to a pin (Future Electronics: 0672-2-15-15-30-27-10-0) with a small insert (track pins; Dataflex: TRP-1558-0000) and were fit into a 10-hole connector, after which the whole assembly was fixed with dental cement to the cranium. Animals were given at least 2 weeks as a recovery period.

\section{Recording, analysis of spectral oscillations and network connectivity}

EEG recordings were derived from six brain regions under vigilance waking condition during the dark circadian phase ${ }^{40,41}$. Additionally, a general motion level was monitored in the home cage by two passive infrared (PIR) detectors placed above each recording cage, and artifactfree waking epochs with low-voltage fast EEG activity, high to moderate EMG and body activities were considered in the analysis. Epochs with high-voltage slow cortical waves in the absence of EMG and locomotor activities were discarded. A notch FIR filter at $50 \mathrm{~Hz}$ was applied to avoid voltage related to power line interferences.

Baseline EEG recordings of 30 min started $2 \mathrm{~h}$ after the light offset to avoid confounding circadian effect on EEG. Afterwards, EEG signals were recorded for $3 \mathrm{~h}$ after vehicle or drug administration $(n=8$ animals for each condition). Continuous EEG and EMG field potentials were acquired at a sampling rate of $512 \mathrm{~Hz}$ with an input range of $\pm 500 \mathrm{mV}$ through a Biosemi ActiveTwo system (Biosemi, Amsterdam, Netherlands), which replaces the conventional ground electrodes by two separate electrodes: the common mode sense (CMS) active electrode and the driven right leg (DRL) passive electrode. This common mode reference for online data acquisition and impedance measures is a feedback loop driving the average potential across the montage close to the amplifier zero. The signals were amplified and analogue band-pass filtered between 1 and $100 \mathrm{~Hz}$ and was digitized with 24bit resolution.

The analysis was performed using a MATLAB toolbox described earlier ${ }^{41}$. Briefly, EEG spectral power density was calculated using a Welch's method with a Hanning window function. Power was expressed as relative power for each frequency over 1-256 Hz. Average relative power in each frequency bin of each location was averaged across animals to obtain the spectrum relative to total power spectrum. For the sake of clarity in presenting this spectral data, graphs only shown the frequency range between 1 and $30 \mathrm{~Hz}$ and inset plots from 30 to $100 \mathrm{~Hz}$.

\section{Coherence}

In order to describe interconnectivity between pairs of EEG electrodes, coherence measure was used, which describe level of connectivity as a value in interval $[0,1]$ (where 1 corresponds to complete perfect relation) for each frequency band $\mathrm{f}$. Coherence is estimated as $\operatorname{Coh}(\mathrm{f})$ $=\left|S_{A B}(f)\right|^{2} /\left(S_{A A}(f) S_{B B}(f)\right)$; where $S_{A B}$ is the crossspectrum between the signals $A$ and $B$ from two different electrodes; $S_{A A}$, is the autospectrum of the signal $A$; $\mathrm{S}_{\mathrm{BB}}$, is the autospectrum of the signal $\mathrm{B}$. Coherence analysis allows assessment of pairwise synchronization of LFP/EEG signals to shed more light onto the interaction between different brain networks.

\section{Electrophysiology \\ In-vivo LTP}

Rats were anesthetized with an intraperitoneal injection of urethane (ethyl carbamate, $1.5 \mathrm{gm} / \mathrm{kg}$, i.p.), and supplemented as necessary $(0.2 \mathrm{~mL} / 100 \mathrm{~g})$ dependent upon the response to a paw pinch. Core body temperature was monitored and maintained at $37^{\circ} \mathrm{C}$ through a heating pad and rectal probe for the duration of the experiments. Once the skull was exposed, two bregma-referenced holes were drilled to insert stimulating twisted-wire bipolar and recording monopolar electrodes constructed from Tefloncoated tungsten wires $(75 \mu \mathrm{m}$ external diameter $)$. Recordings of field excitatory post synaptic potentials (fEPSPs) were made from the stratum radiatum in the CA1 area of the right hippocampal hemisphere in response to stimulation of the ipsilateral Schaffer collateral-commissural pathway.

The electrode implantation sites were identified using stereotaxic coordinates, with the stimulating electrodes $-3.4 \mathrm{~mm}$ posterior to bregma, $-2.5 \mathrm{~mm}$ lateral to midline and $1.9-2.4 \mathrm{~mm}$ ventral, and the recording site located $-4.2 \mathrm{~mm}$ posterior to bregma, $-4 \mathrm{~mm}$ lateral to the midline and $2.5-3.4 \mathrm{~mm}$ ventral. Before the experiment, the correct placement of SC-CA1 implants were finely adjusted by altering the depth of both stimulation and recording electrodes in $10 \mu \mathrm{m}$ increments for optimal field post synaptic potentials (fEPSP) evoking through the oscilloscope. Two epidural screws were inserted in the skull over the cerebellum served, respectively, as the reference and the recording ground. During surgery, all efforts were made to minimize animal suffering. 


\section{HFS for LTP induction}

Stimuli were delivered using a constant current isolator unit (multichannel system MC STG4002). The induced field potential response of the $\mathrm{SC1}$ was passed through the active two electrodes Biosemi amplifier (Differential amplifier, Netherlands) and digitized at $3 \mathrm{kHz}$. At the beginning of each experiment, an input-output (I/O) curve with stimulus at a frequency of $0.033 \mathrm{~Hz}$ and intensities ranging from 1 to 10 Volts was generated for each animal to determine the maximum fEPSP slope, and averaging five responses per intensity, then the intensity of test stimulus was set at a level that evoked an fEPSP slope of $50 \%$ of the maximum was used for all subsequent stimulations.

After the determination of I/O curves, the test stimulation was applied every $30 \mathrm{~s}$ before and after tetanic stimulation. For each time point measured during the experiments, five records of evoked responses at the frequency of $0.033 \mathrm{~Hz}$ were averaged. Baseline activity was measured every $2.5 \mathrm{~min}$ for at least $1 \mathrm{~h}$ to ensure stable baseline. The last $30 \mathrm{~min}$ of the baseline recording (12 time points), immediately after drug application was averaged and used as control for LTP induction. Tetanisation was induced using a high-frequency stimulation (HFS) $200-\mathrm{Hz}$ protocol consisting of square pulses $(0.2$ msec stimulus duration, 10 bursts of 20 stimuli, $2 \mathrm{~s}$ interburst interval) at a stimulus intensity that evoked an fEPSP slope that was approximately $50 \%$ of the maximal response. fEPSP were recorded during $120 \mathrm{~min}$ after HFS to determine possible changes in the synaptic response of SC1 neurons. LTP measurements were derived from field EPSP ratios of the normalized slope average obtained 120-min following HFS divided by the normalized slope average collected $30 \mathrm{~min}$ prior to HFS. Slope of putative fEPSP were measured between the end of stimulus artefact and the trough of the negative peak. The slope of the fEPSP was calculated using a linear fit least square analysis on the $80 \%$ interval between the artefact end and the negative peak. fEPSP slopes were obtained every $2.5 \mathrm{~min}$ as an average of 5 responses at $0.033 \mathrm{~Hz}$ and were then expressed as percentage change from baseline (defined as the last $30 \mathrm{~min}$ prior tetanisation). LTP was defined as an increase in fEPSP slope that is maintained above $120 \%$ relative to baseline for $2 \mathrm{~h}$ following HFS administration. At the end of the electrophysiological study, three 30-s electrical pulses of $500 \mu \mathrm{A}$ were delivered to produce a lesion at the end tip of the stimulation and recording electrodes and brains were harvested for histological verification of electrodes placement. Brain sections $(20 \mu \mathrm{m})$ were examined using a light microscope. Animals with incorrect electrode placement were excluded from the study.

\section{Drugs}

The GlyT1i (SSR504734), D-serine, and rolipram were purchased from Sigma Aldrich. SSR504734 (2.5, 10 and $40 \mathrm{mg} / \mathrm{kg}$ ) was formulated in $10 \% \mathrm{CD}+1 \mathrm{HCl}+\mathrm{NaCl}$. Rolipram (1, 3 and $10 \mathrm{mg} / \mathrm{kg}$ ) was formulated in $10 \% \mathrm{CD}$ $+1 \mathrm{HCl}+\mathrm{NaCl}$. D-serine $(20,80$ and $320 \mathrm{mg} / \mathrm{kg}$ ) was dissolved in $\mathrm{NaCl}+\mathrm{H} 2 \mathrm{O}+\mathrm{NaOH}$. Drugs were administered subcutaneously in a volume of $1 \mathrm{ml} / 100 \mathrm{~g}$ body weight.

\section{Data analysis \\ EEG}

Result for EEG spectrum metrics were calculated for each frequency bin and were expressed as relative total power spectra during $3 \mathrm{~h}$ following the administration of test drugs. Animal numbers were chosen to ensure adequate statistical power comparable to previously published papers. Data distribution was assumed to be normal and were presented as mean values with 95\% confidence intervals (CI). One-way ANOVA followed by Dunnett's post hoc test were used to assess difference in means between vehicle and different drug doses for EEG power and coherence measures while considering the heterogeneity between animals. ANOVA results are reported in figures' captions, and mean data are visualised as box plots with significant differences based on post hoc analysis indicated by asterisks (" $p$-value $<0.05$, ** $p$-value $<0.01)$.

\section{LTP}

In vivo electrophysiology fEPSP responses recorded after application of drugs were expressed as percentage of change from baseline. The slope of the fEPSP was calculated using a linear fit least square analysis on the $80 \%$ interval between the artefact end and the negative peak. fEPSP slopes were obtained every $2.5 \mathrm{~min}$ as an average of 5 responses at $0.033 \mathrm{~Hz}$ and were then expressed as mean percentage change from baseline (defined as the last 30 min prior tetanisation) \pm SEM. Firstly, in order to assess longitudinal changes after application of HFS, mixed-effect modelling was applied. Time after HFS was $\log$-transformed as $t_{\text {new }}=\ln \left(1+t_{\text {old }} / 5\right)$, where $t_{\text {old }}$ is a real time expressed in minutes. This transformation allowed to linearize the data. As a next step, fEPSP relative to baseline (\%) variable was modelled as $t_{n e w}{ }^{*}$ condition + (1|animal), where condition variable is categorical variables describing vehicle and different drug concentrations. Effect of condition variable on intercept (to assess initial differences in fEPSP responses) and slop (to assess differences in attenuation of fEPSP responses in time) of the model were tested. Of primary interest there were differences between vehicle and drug. Secondly, in order to 
understand difference between conditions at each time point after HFS, repeated measures analysis of variance (ANOVA) followed by a post-hoc test (Dunnett's test) were used. $p<0.05$ was considered statistically significant.

\section{Results \\ Effects of SSR504734, D-serine and rolipram on network oscillation and connectivity}

Previous reports reveal the presence of two different theta (slow (4-6) and fast $(6.5-8 \mathrm{~Hz})$ and gamma bands (slow $(30-50 \mathrm{~Hz})$ and fast $(50-100 \mathrm{~Hz})$ ). Both rhythms are involved in the communication between CA1 and CA3 and between CA1 and entorhinal cortex ${ }^{40,42,43}$ and showed sensitivity to pharmacological treatment ${ }^{40}$. Therefore, we evaluated LFP power in slow, and fast theta-gamma rhythms of the CA1-CA3-frontal circuit during treatment with D-serine, SSR504734 and rolipram.

No major effect was observed on slow and high theta or gamma spectra after D-serine treatment. No frequency band showed consistent changes between 0 and $100 \mathrm{~Hz}$ across regions from pre-injection levels and compared to vehicle (Fig. 1). In addition, there was no site-pair by frequency changes for peak coherence after the administration of different does of D-serine (Fig. 4 only mean peak slow theta coherence was displayed over the $3 \mathrm{~h}$ period following the administration).

In contrast, LFPs recorded after SSR504734 and rolipram were associated with similar pattern of power and peak coherence changes. The EEG of rats shifted to continuous synchronized activity to slow theta oscillations at the CA1 network after the administration of both drugs in the model (Figs. 2 and 3). Rolipram further synchronized slow theta at the CA3 level (Fig. 3), while no major changes were observed in the activity of other frequency bands in CA1-CA3 network. SSR504734 at the higher dose yielded additional patterns of activity consisted with enhancing effect on frontal-CA1 network gamma power (Fig. 3). Coherence of both SSR504734 and rolipram peaked at the slow theta frequency in the CA1-CA3 network (Fig. 4 middle and right panel, respectively).

To assess whether changes in EEG slow theta activity $(4-6.5 \mathrm{~Hz})$ oscillations were associated with changes in motor behaviour following different treatments, the time course of motor activity at different time points revealed a reduction in activity levels (Fig. 5).

Next, we examined whether the observed differences in hippocampal oscillation are associated with changes in synaptic plasticity.

\section{Effects of exogenous D-serine on LTP}

The effect of exogenous D-serine $(20,80,320 \mathrm{mg} / \mathrm{kg})$ was investigated on LTP induction and maintenance. Baseline input/output (I/O) curves for fEPSP slopes were not significantly different confirming that the SC fibres in all dose groups had similar basal synaptic transmission (Fig. 6). D-serine had no effect on basal synaptic activity.

Mixed-effect model revealed significant effect of Dserine after the HFS tetanisation on short term potentiation "STP" (model's intercept: $p<0.001$, and each concentration of drug was significantly different from vehicle $[p<0.001])$ and inhibition effect on LTP in time (model's slope: $p<0.001$, and each concentration of drug, except for $40 \mathrm{mg} / \mathrm{kg}$, was significantly different from vehicle $[p<$ $0.001])$. Absence of significant difference of the $40 \mathrm{mg} / \mathrm{kg}$ injection on model's slope $(p=0.77)$ characterizes, that LTP after vehicle and $40 \mathrm{mg} / \mathrm{kg}$ injections were inhibited at the same rate.

More precisely, after HFS tetanisation, D-serine impaired STP $(-7.2 \%,-16.1 \%$ and $-17 \%: p=0.02$, respectively) and the expression to LTP (over $2 \mathrm{~h}$ ). Further analysis into the last period of the recording session revealed a significant inhibitory effect on LTP maintenance, particularly with the higher dose (90-120 min post-HFS, $-18 \%: p=0.03$ ) (Fig. 6).

\section{Effects of SSR504734 on LTP}

The effect of increased glycine levels via reuptake inhibition on LTP expression was investigated. I/O curves were not significantly different confirming that the SC fibres in all dose groups were of a similar excitability (Fig. 7). SSR504734 had no influence on basal synaptic transmission.

Mixed-effect model did not reveal significant effect of SSR504734 after the HFS tetanisation on STP (model's intercept: $p=0.058$ ), but the effect on enhancement of LTP in time was significant (model's slope: $p<0.001$ ).

More precisely, SSR 504734 at $10 \mathrm{mg} / \mathrm{kg}$ failed to enhance the slope of fEPSPs above vehicle levels during the entire recording session of $2 \mathrm{~h}$ post-treatment. However, there was a significant effect of SSR504734 at the dose of $40 \mathrm{mg} / \mathrm{kg}$ on LTP maintenance as revealed during the last period of the recording session after HFS (90-120 min post-HFS, $+41 \%: p=0.001)$.

\section{Effects of rolipram on LTP response}

The effect of increasing cAMP levels with the selective blocker of PDE4 reuptake inhibition on LTP expression was studied. The results showed no significant difference between group I/O curves confirming there was no difference in the excitability SC-CA1 synapses in the study groups (Fig. 8). Similarly, basal synaptic transmission was not affected by application of rolipram.

Mixed-effect model revealed significant effect of rolipram after the HFS tetanisation on STP (model's intercept: $p<0.001$ ) and enhancement effect on LTP in time (model's slope: $p<0.001$ ).

Detailed analysis of difference driving above's significance results showed, that the dose of $3 \mathrm{mg} / \mathrm{kg}$, 


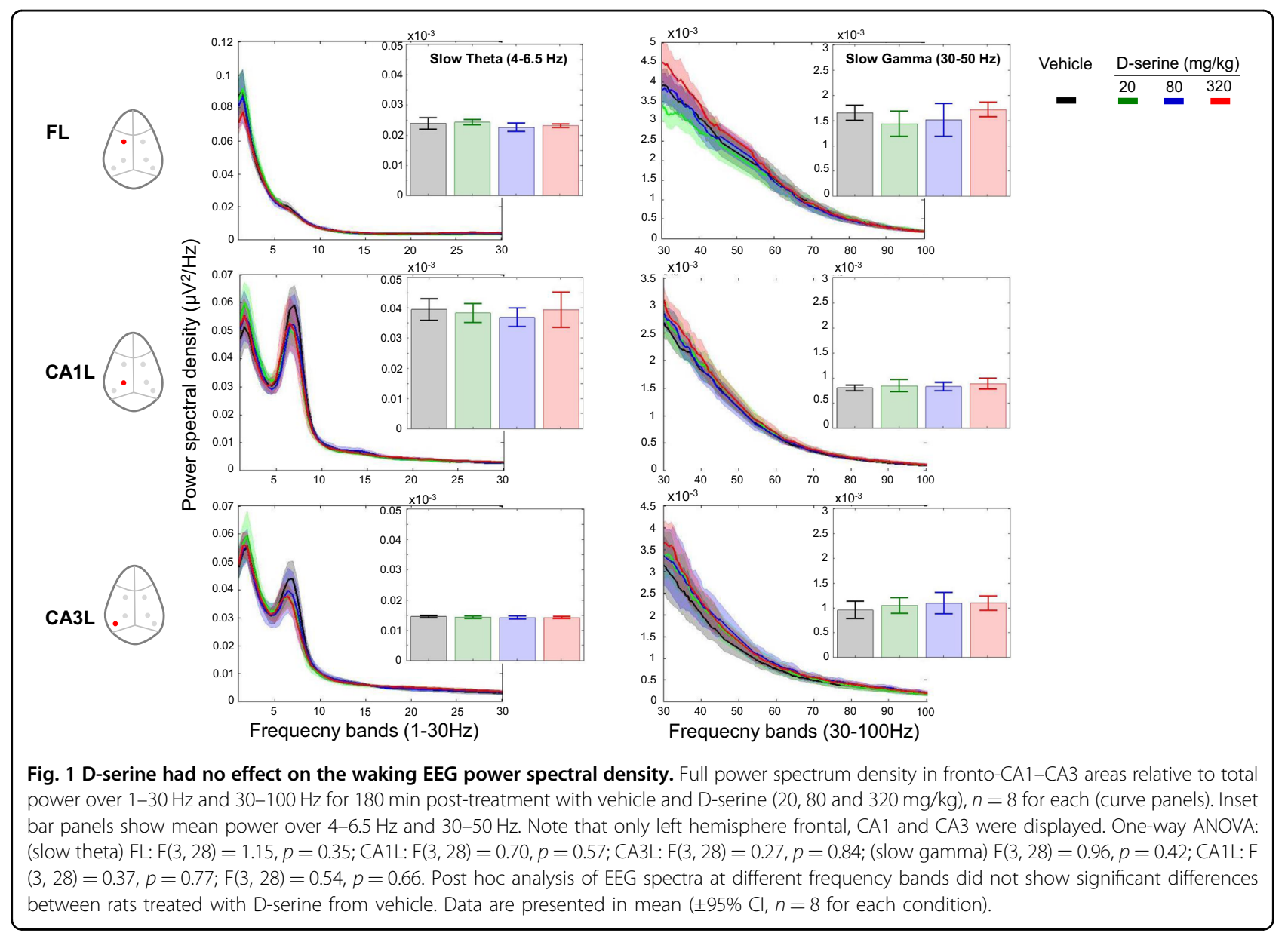

rolipram led to the increased STP $(+25 \%, p=0.04$, Fig. 8), however, this transient potentiation did not express into LTP. At the lowest dose of $1 \mathrm{mg} / \mathrm{kg}$, rolipram had no significant effect on STP and the expression into LTP as compared to vehicle (Fig. 8).

\section{Discussion}

In the present study, we sought to define patterns of network oscillatory activities and plasticity amongst anatomically and functionally connected brain region after modulation of both NMDA and cAMP signalling. Both SSR504734 and rolipram enhanced network slow theta oscillations, connectivity in the CA1-CA3 circuit. SSR504734 enhanced LTP response, whereas the plasticity was short-lived to STP with rolipram. Unlike glycine, increased levels of D-serine had no effect on network oscillations and limits the induction and expression of LTP.

Neural oscillations are critical mechanisms allowing dynamic coupling of intra- and inter-regional brain regions during information processing ${ }^{44,45}$. For instance, theta oscillatory rhythm derived from concerted generators including cholinergic and GABAergic, as well as glutamatergic networks in different locations, plays a key role in the function of the hippocampus and associated cortical networks ${ }^{46}$. Afferent cholinergic and glutamatergic input from the medial septum-diagonal band of Broca, as well as from CA3 and entorhinal cortex provide support for theta oscillations at the CA1 circuit $^{46}$. Modulation of the glutamatergic NMDA signalling through local modulation of septal circuit mediates the generation of hippocampal theta rhythm ${ }^{47}$. Theta oscillations may facilitate synchrony between hippocampus and prefrontal cortex network required for learning and memory consolidation ${ }^{48}$.

Gamma oscillations $(30-100 \mathrm{~Hz})$ are prominent in the cortical-hippocampal network and have been shown to appear during a variety of memory tasks in rats, monkeys, and humans ${ }^{43,49}$. Gamma rhythms occur as two distinct variants that are thought to route different streams of information entering hippocampal subfield $C A 1^{42,50}$. Slow gamma $(30-50 \mathrm{~Hz})$ may promote direct inputs from CA3 to $\mathrm{CA1}$, which is believed to be associated with memory retrieval $^{51,52}$. Fast gamma $(50-100 \mathrm{~Hz})$ may facilitate direct transmission from the medial entorhinal cortex that transmit ongoing spatial information ${ }^{51,53}$.

Functional roles of theta and gamma oscillations in mnemonic processes has been established ${ }^{54}$, and while 


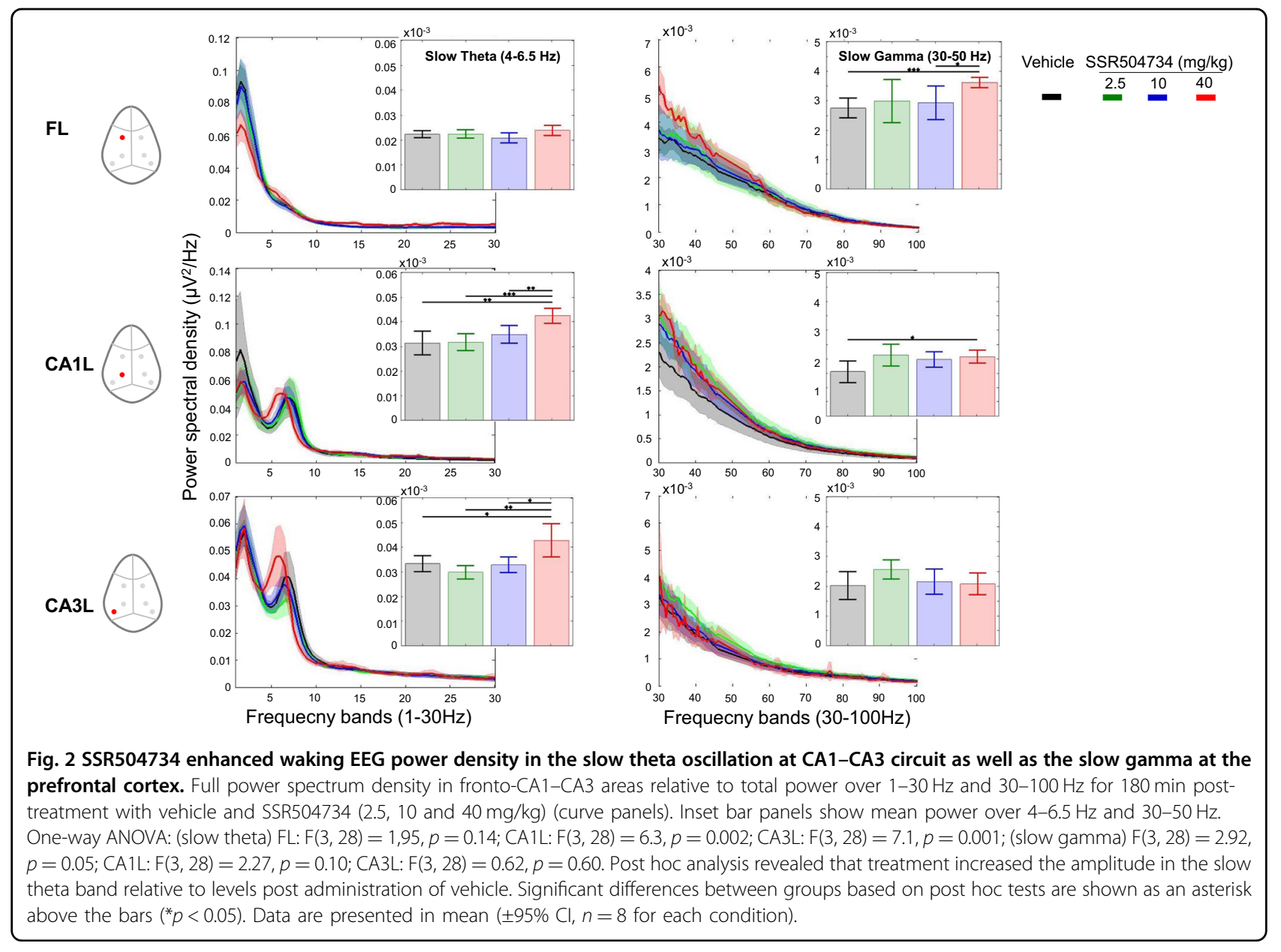

disruption of this oscillatory rhythms has been associated with cognitive deficits described in psychiatric and neurodegenerative disorders ${ }^{55,56}$. Pharmacological and several new therapeutic techniques, such as transcranial magnetic stimulation, transcranial direct current stimulation, and closed-loop stimulation, have profound direct and indirect effects on ongoing oscillatory activity in the brain ${ }^{57}$, and restoration of theta and gamma like rhythmicity restores learning and memory capabilities in rats ${ }^{58-60}$.

\section{SSR504734 and rolipram but not D-serine enhanced network oscillations and connectivity}

Abundant evidence points to the importance of NMDA receptors in patterning neuronal networks and synaptic transmission. Positive modulation of the co-agonist binding site on the NMDA receptor has been proposed as a novel therapeutic approach to overcome negative symptomatology and cognitive dysfunction seen in those diseases ${ }^{61}$. Glycine and D-serine are endogenous ligands to the NMDA modulatory site $^{62}$, and ligands modulating NMDA receptor transmission, have being developed such as GlyT-1 inhibitors to potentially elevate brain glycine or targeting enzymes, such as d-amino acid oxidase (DAAO) to slow the breakdown and increase the brain level of D-serine ${ }^{63,64}$. In the present study we further evaluated effects of the glycine inhibitor, exogenous D-serine or modulating cAMP levels on synaptic network oscillations and plasticity. The oscillatory activity across the CA1 network of structures studied in the present work was dominated by changes in the slow theta rhythm and related coherent activity. Thus, indirect modulation of NMDA signalling through inhibition of the GYT1 may support the hypothesis that the release of endogenous Dserine from astrocytes did not saturate and, therefore, enough to generate and entrain synchronized theta rhythm in CA1-CA3 network during information processing. However, exogenous supply of D-serine failed to promote similar changes in network oscillation and connectivity, likely because D-serine levels might have reached saturation and therefore shunt down synchronized theta rhythm. In addition, SSR504734 enhanced slow gamma at the frontal and CA1 structures, which of rhythm has been hypothesized to promote memory retrieval.

Therefore, the evoked slow theta and gamma network oscillations at the CA1 and frontal networks, respectively 

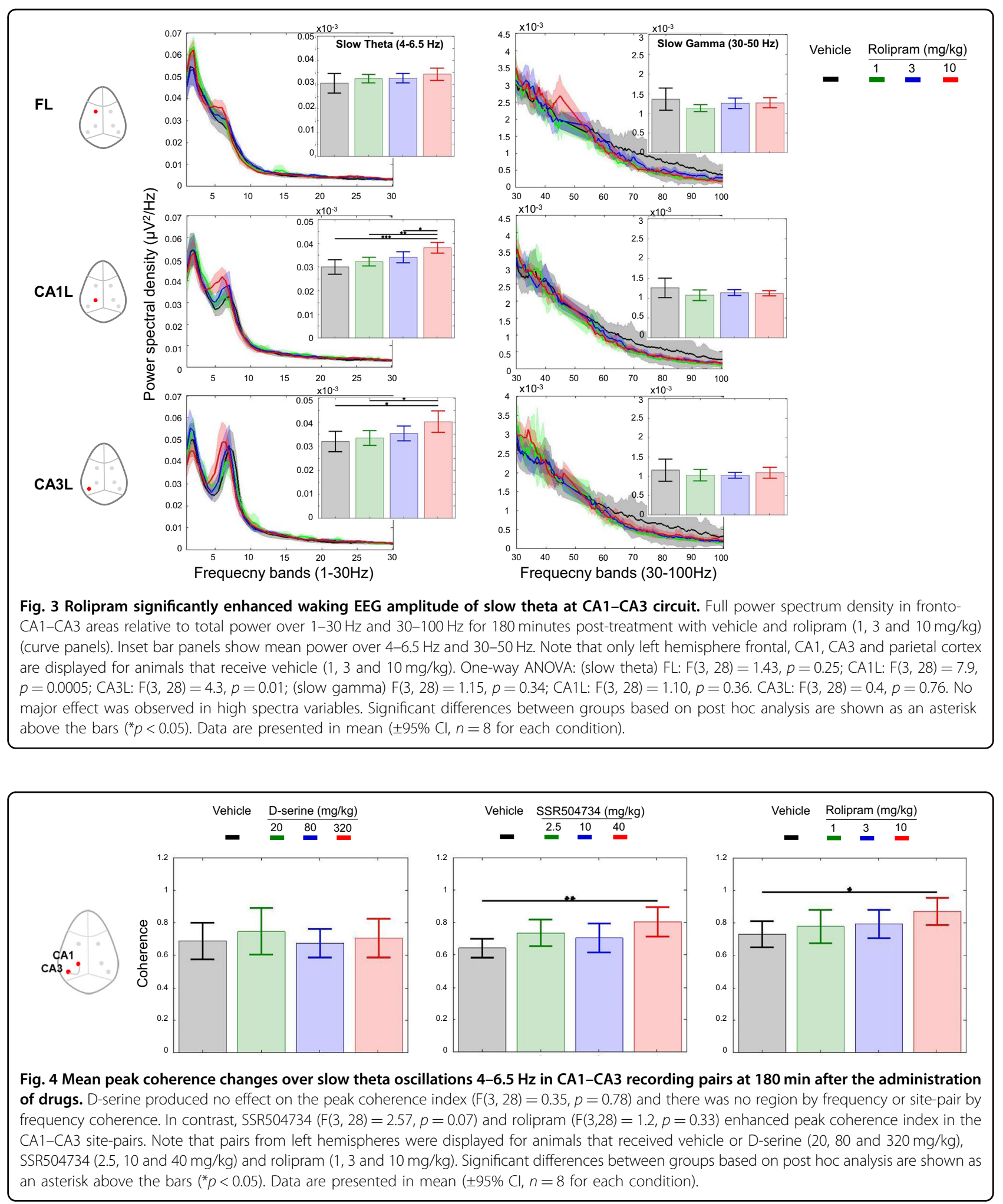

suggests a positive modulation effect of the glycine site of the NMDA receptor on attentional processing and memory operations phases.
Alterations in cAMP signalling are thought to contribute to neurocognitive and neuropsychiatric disorders. Phosphodiesterases play an essential role in 


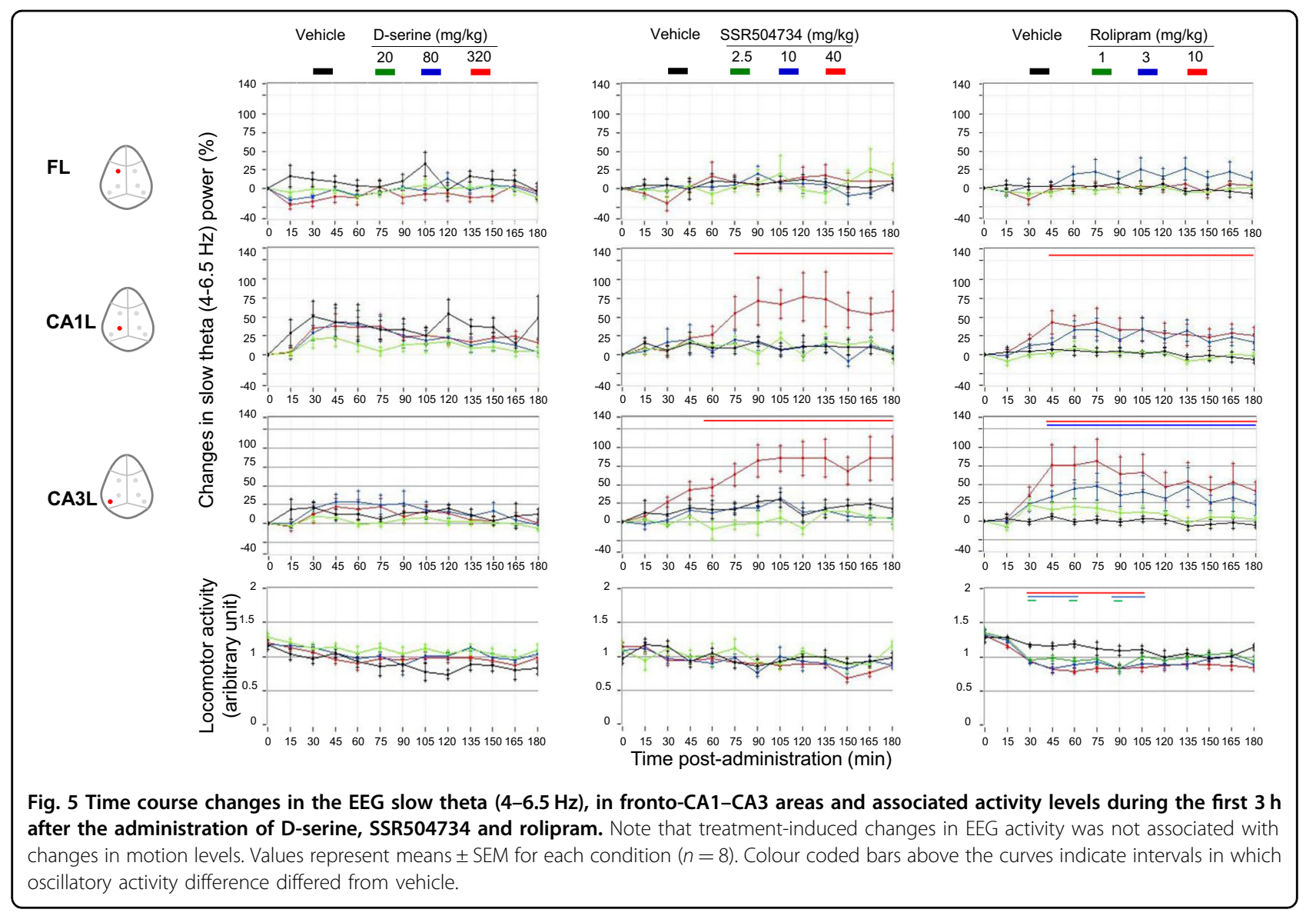

orchestrating the compartmentalized degradation of cAMP, leading to local changes in cAMP signalling in specific subcellular domains in the cell ${ }^{65}$. The cAMP signalling pathway is a second messenger that has a key role in several intracellular cascades, including the cAMP/protein kinase A (PKA)/cAMP response element-binding protein (CREB) pathway which is critically involved in learning and memory ${ }^{5}$. Changes in cAMP levels has been shown to regulate theta activity, and rolipram administered intravenously evoked an arousal EEG pattern period (low amplitude fast waves) in the cortical EEG and synchronization of the hippocampal theta waves with increased voltages ${ }^{66}$. In the amygdalo-hippocampal pathways, an increase in intracellular cAMP concentration facilitates regular firing and oscillatory activity at the theta frequency range $^{67}$, supporting synaptic signal transfer between those functionally connected neuronal populations during retrieval of conditioned fear. Disruption of theta activity results in spatial memory deficits, whereas the restoration of theta-like rhythmicity reverse learning deficits in rats ${ }^{59,60}$. In the present work, the enhanced slow theta activity confirmed the potential positive modulatory effect of rolipram on neural networks.

\section{Glycine transporter inhibitor and rolipram, but not D- serine, enhanced in vivo plasticity LTP and glycine}

Activity-dependent synaptic plasticity, such as NMDARdependent LTP has been proposed as a cellular mechanism underlying learning and memory in the brain ${ }^{68,69}$. The GlyT1 antagonist NFPS increased NMDAR channel opening in a dose-dependent manner in Sprague-Dawley prefrontal cortex slices ${ }^{70}$. Similarly, the antagonist CP-802,079 enhanced LTP induced by HFS in hippocampal slices ${ }^{14}$. However, the effects of both these antagonists appear irreversible. Depoortère et $\mathrm{al} .{ }^{71}$ first reported on the neurochemical, electrophysiological and pharmacological characteristics of the selective, reversible GlyT1 inhibitor SSR504734. The compound enhanced in a concentration-dependent manner the NMDA component of CA1 excitatory postsynaptic currents therefore further confirming the role of the GlyT1 in NMDA excitability. In the present study, SSR504734 enhanced the LTP expression at the highest dose of $40 \mathrm{mg} /$ $\mathrm{kg}$ for the entire 2-h post-tetanisation recording period. The significantly enhanced and enduring LTP is likely the result of increased NMDAR channel openings ${ }^{70}$ as this would cause a larger postsynaptic influx of $\mathrm{Ca}^{2+}$ and activation of LTP maintenance mechanisms. 

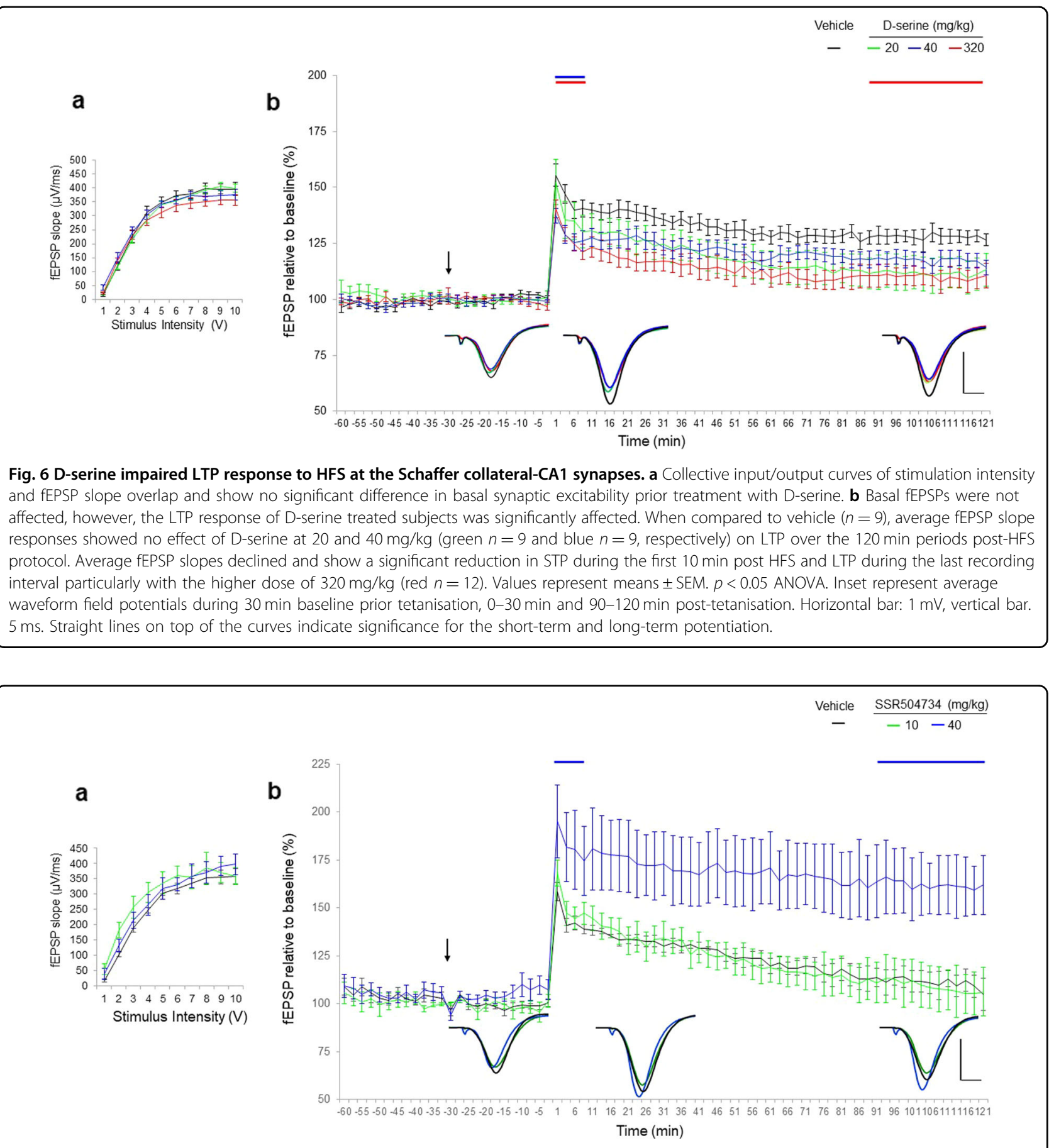

Fig. 7 SSR504734 enhanced LTP response to HFS at the Schaffer collateral-CA1 synapses. a Collective I/O curves of stimulation intensity and fEPSP slope overlap and show no significant difference in basal synaptic excitability prior treatment with SSR504734. b Average fEPSP slope responses show that SSR504734 at the dose of $10 \mathrm{mg} / \mathrm{kg}$, had no effect on the induction of LTP (green, $n=5$ ) as compared to vehicle (black, $n=5$ ). At the dose of $40 \mathrm{mg} / \mathrm{kg}$ (blue, $n=6$ ), SSR504734 enhanced LTP as compared to vehicle. Values represent means \pm SEM. Values represent means \pm SEM. $p<0.05$ ANOVA. Inset represent average waveform field potentials during $30 \mathrm{~min}$ baseline prior tetanisation, 0-30 min and 90-120 min post-tetanisation. Horizontal bar: $1 \mathrm{mV}$, vertical bar. $5 \mathrm{~ms}$. Straight lines on top of the curves indicate significance for the short-term and long-term potentiation.

The lower dose of SSR504734 given, $10 \mathrm{mg} / \mathrm{kg}$ had no effect on LTP as compared to the vehicle. Microdialysis studies in the prefrontal cortex and nucleus accumbens revealed a significant increase in the extracellular glycine concentrations for $45-180 \mathrm{~min}$ after the application of SSR504734 at the dose of $10 \mathrm{mg} / \mathrm{kg}^{71,72}$. According to this 


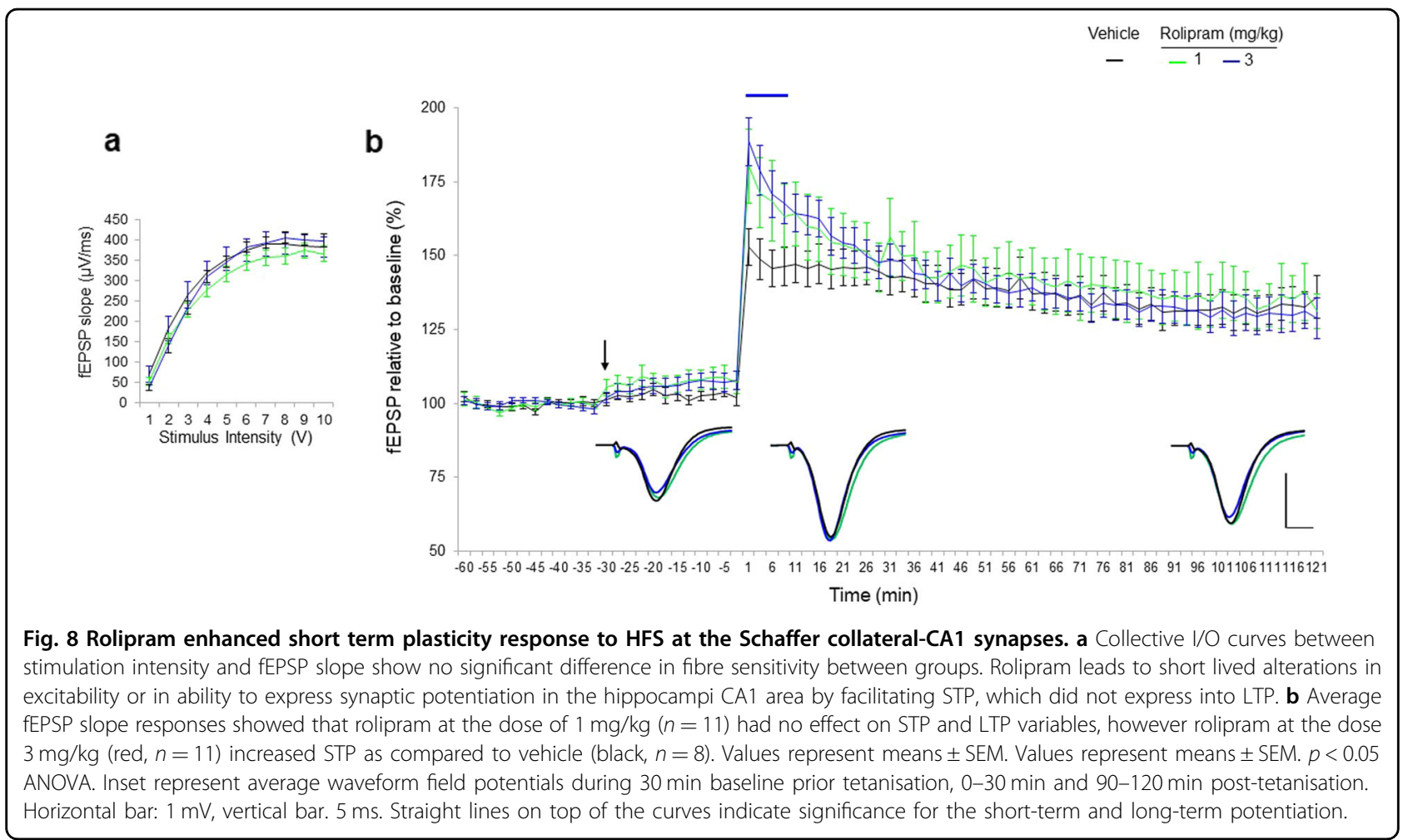

time frame, the HFS in this study fell $15 \mathrm{~min}$ before synaptic glycine concentrations were increased. It is, therefore, possible that SSR504734 $(10 \mathrm{mg} / \mathrm{kg})$ successfully increased glycine concentrations in this study. However, as this would not have occurred prior to or during the tetanisation, the extra glycine at the synapse following GlyT1 inhibition would not have been able to enhance NMDAR excitability before administration of the HFS. As a result, the NMDAR-dependent LTP of CA1 would not have been enhanced by the GlyT1 inhibition. The $40 \mathrm{mg} / \mathrm{kg}$ dose may also have a faster time frame for glycine concentration increase following injection due to the higher concentration of compound available. Extracellular increase in glycine concentration has not been investigated at doses above $10 \mathrm{mg} / \mathrm{kg}$ of SSR $504734^{71,72}$. A subsequent microdialysis study could confirm the hypothesis that HFS was administered before peak glycine levels with the $10 \mathrm{mg} / \mathrm{kg}$ dose and that this effect was shifted rightwards following $40 \mathrm{mg} / \mathrm{kg}$.

SSR504734 has also been investigated in mice performing in an operant delayed alternation cognitive $\operatorname{task}^{63}$. Success in the task, which relies on working memory, became more difficult with longer time intervals and delays above $8 \mathrm{~s}$ between trials proved to be challenging. Animals treated with the dose of $30 \mathrm{mg} / \mathrm{kg}$ successfully completed tasks with intervals of $12-18 \mathrm{~s}$, intervals at which control animals could no longer perform above chance levels. At intervals of 12 s, SSR504734
(30 mg/kg) enhanced the percentage of correct choices as did $10 \mathrm{mg} / \mathrm{kg}$, however, this was not a significant effect. Other studies that used SSR504734 showed efficacy in behavioural studies with doses higher than $10 \mathrm{mg} / \mathrm{kg}^{73,74}$. Therefore, the present results support the earlier behavioural studies. SSR504734 $1(0 \mathrm{mg} / \mathrm{kg})$ may enhance glycine levels but not sufficient to overcome the effect of other factors in an intact brain. Indeed, the other NMDAR coagonist D-serine exhibits higher affinity for the strychnine-insensitive binding site ${ }^{9}$. This binding competition could prevent intermediately increased glycine levels from having a detectable effect on LTP or behavioural response. The results of this work suggest that reuptake inhibition with SSR504734 and the two GlyT1 inhibitors NFPS ${ }^{12}$ and CP 802,079 ${ }^{14}$ did indeed lead to increased synaptic levels of glycine, therefore increasing its role as a facilitator of NMDAR excitability and the enhancement of LTP following HFS. The results suggest a beneficial effect of GlyT1 inhibitors on hippocampal-dependent forms of memory deficient, and further provide a compelling rationale for using GlyT1 inhibitors to indirectly potentiate NMDA receptor functions.

\section{LTP and D-serine}

Endogenous D-serine can be released in an activitydependent manner and, in turn, contributes to the induction of LTP and LTD ${ }^{19,75}$. D-serine is a more potent 
agonist of the NMDAR than glycine ${ }^{9}$, and D-serine is moderately better than glycine in penetrating the blood-brain barrier when administered systemically, therefore, it was expected that the high doses of D-serine given in this experiment would potentiate the LTP response. However, this was not the case with the middle dose $(40 \mathrm{mg} / \mathrm{kg})$ had a slightly depotentiation effect on LTP levels and the highest dose $(320 \mathrm{mg} / \mathrm{kg})$ decreasing LTP response. Whilst some groups have reported enhancing LTP with D-serine application ${ }^{16,76}$, the consensus in the literature is that exogenous D-serine has no effect on LTP in wild-type animals even though its increased NMDAR-mediated post-synaptic responses in hippocampal slices ${ }^{77-79}$. D-serine treatment alone has no effect on LTP induction, while it decreased the basal glutamatergic neurotransmission, which weakens the efficacy of the agonist on synaptic plasticity and depresses basal AMPA receptor-mediated neurotransmission in young animals. This property may, therefore, limit the potency of the agonist to increase the magnitude of synaptic plasticity, especially in aged rats as AMPARmediated neurotransmission was reduced in these animals ${ }^{80,81}$. D-serine had no effect on spatial learning and memory per $\mathrm{s}^{82}$, whereas exogenous application of $\mathrm{D}$ serine has however been shown to restore potentiation in aged animal models of decreased D-serine concentrations ${ }^{77}$ or in pharmacologically induced glial metabolism disruption $^{79}$. Another difference that may explain discrepancies of results may be related to stimulation protocols used to induce LTP. In both groups that showed an enhancement of LTP under control conditions have used theta-burst protocol, which may activate different signalling pathways as compared to HFS as well as induce a different pattern of $\mathrm{Ca}^{2+}$ signalling ${ }^{83}$. However, plasticity studies that used HFS protocols did not reveal significant increase of the LTP response following D-serine, which may cause endogenous D-serine levels to saturate following tetanisation and shunts inhibition of afferent inputs which thus display a depression (an LTD-like effect) instead of an LTP at the soma ${ }^{78}$. This may explain a negative trend in basal synaptic activity and the LTP responses that were seen in this study using young, wild type rats and HFS protocol.

The novel finding reported here is that the highest dose of exogenous D-serine reduced synaptic potentiation below control levels, which could lead to receptor internalization. Both D-serine and glycine binding have been suggested to prime the NMDA receptor for clatherindependent endocytosis upon glutamate binding and receptor activation in hippocampal cells ${ }^{84}$. In this case, the high dose of $\mathrm{D}$-serine would increase the number of receptors primed for internalisation. Upon application of the HFS, which would cause a large glutamate release, postsynaptic depolarisation would cause NMDA receptor endocytosis. If an enough NMDA receptor were internalised this would reduce the levels of signalling $\mathrm{Ca}^{2+}$ and therefore reduce the activation of LTP signalling mechanisms. As the study by Nong et al. ${ }^{84}$ focussed more prominently on the role of glycine in NMDA receptor internalisation, further immunocytochemical assays using D-serine could strengthen this hypothesis.

Another possibility for the decreased LTP response after high dose of D-serine may result in synaptic excitotoxicity. Indeed, high concentrations of this amino acid have been measured in pathological, neurotoxic states such as cerebral ischemia ${ }^{85}$. In AD, amyloid- $\beta$ has been shown to induce D-serine release from microglia leading to neurodegeneration ${ }^{86}$. It has also been reported that exogenous D-serine has a dose-dependent bell-shaped effect on LTD magnitude ${ }^{75}$, which could reflect changes in the LTD/LTP threshold as set out in the Bienenstock, Cooper, and Munro (BCM) model. Accordingly, the high level of D-serine $320 \mathrm{mg} / \mathrm{kg}$ may be closer to the LTP threshold than lower or higher doses of exogenous D-serine and inducing a lower level of LTP than under vehicle or $320 \mathrm{mg} / \mathrm{kg}$ conditions. However, to our knowledge it has not been investigated whether this is also the case for LTP as single concentrations of D-serine were generally used in previous studies.

Whilst both glycine and D-serine directly modulate the excitability of NMDA receptors and enhance NMDARmediated synaptic transmission, clinical trials involving direct administration of both amino acids produced mixed results in improving cognitive impairments in schizophrenic patients ${ }^{87}$. The present study provides evidence that D-serine controls NMDAR-dependent LTP, whilst glycine influence neurotransmission at a different level, by activating extrasynaptic glycine receptors distributed along the apical dendrite. Future studies will evaluate mechanistic approaches targeting D-serine modulatory sites, for example by inhibition of the enzyme d-amino acid oxidase (DAAO), which slows the break-down of D-serine, or by its transporter, the alanineserine-cysteine-1 (Asc-1).

\section{LTP and rolipram}

Alterations in the activity of PDE4 has been associated with cellular mechanisms underlying structural and synaptic damage in experimental models of mood and neurological diseases ${ }^{6}$. Pharmacological inhibition of PDE4 activity promotes synaptic plasticity and memory ${ }^{88}$. Mice lacking all PDE4D isoforms display either memory enhancements or impairments, depending on the task used $^{17}$. The PDE4 selective inhibitor, rolipram, prevents memory deficits associated with sleep $\operatorname{loss}^{89}$, aging ${ }^{90}$, muscarinic or NMDA receptor blockade ${ }^{39}$, and mouse models of Alzheimer's disease ${ }^{91}$. LTP can be induced in the SC-CA1 synapse of the hippocampus by stimulation 
in the theta frequency range $(5-12 \mathrm{~Hz})$, an effect that depends on activation of the cAMP pathway ${ }^{92}$. The amyloid beta-induced inhibition of LTP in slices was reversed following direct application of rolipram ${ }^{93}$.

In this study, an enhancement of early phase of LTP was observed following the administration of rolipram at $3 \mathrm{mg} / \mathrm{kg}$. This would appear contradictory with the hypothesised role of the cAMP/PKA pathway in late memory consolidation and late-LTP ${ }^{36}$. Late-LTP is a more persistent and robust form of LTP lasting for 8-10 h that requires PKA activation for protein synthesis and to facilitate LTP maintenance ${ }^{94}$. Late-LTP can be induced using a strong tetanisation procedure such as the repetition of HFS trains ${ }^{94}$. Similar late-LTP levels have been recorded in vitro and in vivo using application of rolipram to enhance cAMP levels ${ }^{39,88}$. Wiescholleck and ManahanVaughan $^{39}$ reported that this dose in vivo caused a transient chemically induced potentiation lasting an hour without tetanisation. However similar doses of rolipram in vitro show an effect on basal synaptic transmission and have been reported to have a negative impact on LTP perhaps through a toxic effect ${ }^{88}$. The amplification of transient cAMP by rolipram and its impact on LTP levels is sensitive to the time at which the HFS protocol is administered $^{88}$. The enhancing effect of rolipram was only seen when the hippocampal slice was perfused with the compound during tetanisation, however, LTP was no different from saline if the perfusion occurred after HFS ${ }^{88}$.
Most likely the highest dose used in this study transiently amplified the cAMP to elicit a potentiation but not sufficiently to fully activate CREB signalling or AMPA insertion at the synapse ${ }^{95}$. The enhancement of LTP by rolipram, although transient, shows that the HFS model is responsive to enhanced cAMP levels following the inhibition of PDE4. The cAMP/PKA path is not the only signalling pathway regulated by the PDEs; cGMP and the related PKG also activate the transcription factor CREB, which may be involved in earlier LTP and memory consolidation to the cAMP pathway ${ }^{36}$. Therefore, a combined enhancement of both CAMP and cGMP could activate mechanisms whilst also inducing the protein processes necessary for facilitating late-LTP.

The present study provides evidence that the glycine modulatory site was required for the induction of NMDAR-dependent LTP and connectivity whilst exogenous D-serine negatively influenced neurotransmission. Unlike glycine, increased level of D-serine does limit the induction and expression of LTP in the rat CA1. We hypothesized that high levels of D-serine might results in a shunt of synaptic inputs to downregulate NMDARs currents and eventually leading to synaptic depression after application of HFS at the somatic level (Fig. 9). Our observations that rolipram elicited a transient induction of short-term form of potentiation that was not facilitated into late phase of LTP, raises the likely possibility that transiently increased cAMP levels immediately before

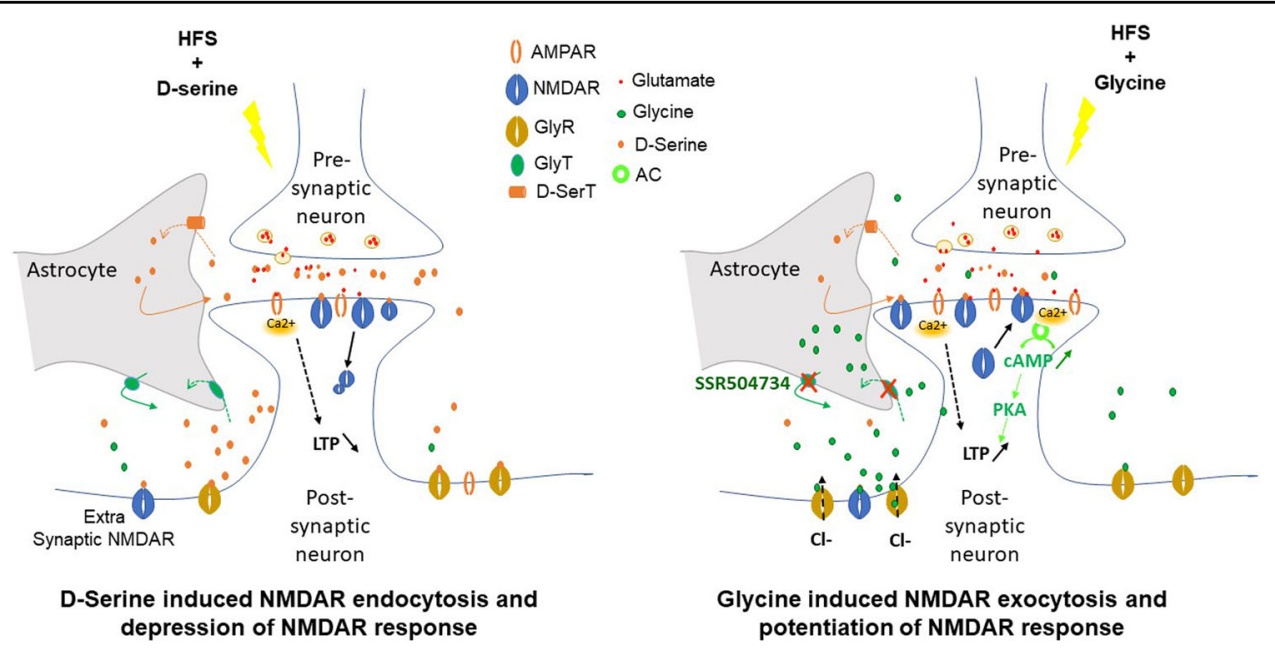

Fig. 9 Schematic depicting the relationship between changes in the levels of D-serine and glycine with synaptic response at the level of NMDA receptors. Glutamate can be released as a gliotransmitters by either $\mathrm{Ca}^{2+}$-dependent vesicular release or efflux from transporters or largeconductance ion channels. Presynaptic glutamate release triggers postsynaptic activation through the binding to membrane NMDA receptors. In the synaptic cleft, glutamate can be taken up by transporters located in astrocytes and converted to glutamine and GABA. Glutamate binds to AMPA receptors on astrocytes, stimulates the release of D-serine, which is synthesized from L-serine via serine racemase in astrocytes. D-serine can be released via either vesicular or non-vesicular mechanisms and activate neuronal NMDA receptors. Under HFS, the increased glutamatergic levels in the synaptic cleft and binding of released D-serine to glycine modulatory sites on the NMDA receptors lead to overactivation of postsynaptic neurons through upregulated NMDA and mGluR receptors, which may result in endocytosis of NMDA receptors and depression of NMDAR signalling and LTP response. GlyT-1 inhibitors increase extracellular glycine levels by inhibiting GlyT-1 in glial cells, and increased glycine levels elicits NMDAR exocytosis, and stimulation of glycine modulatory sites on the NMDA receptors to potentiate NMDA receptor signalling and LTP response. 
tetanisation attenuated the molecular machinery involved in mediating the late phase of LTP in the SC-CA1 synapses.

\section{Conflict of interest}

The authors declare that they have no conflict of interest.

\section{Publisher's note}

Springer Nature remains neutral with regard to jurisdictional claims in published maps and institutional affiliations.

Received: 8 February 2020 Revised: 21 May 2020 Accepted: 26 May 2020 Published online: 18 June 2020

\section{References}

1. Rebola, N., Srikumar, B. N. \& Mulle, C. Activity-dependent synaptic plasticity of NMDA receptors. J. Physiol. 588, 93-99 (2010).

2. Dauvermann, M. R., Lee, G. \& Dawson, N. Glutamatergic regulation of cognition and functional brain connectivity: insights from pharmacological, genetic and translational schizophrenia research. Br. J. Pharm. 174, 3136-3160 (2017).

3. Sanderson, T. M. \& Sher, E. The role of phosphodiesterases in hippocampal synaptic plasticity. Neuropharmacology 74, 86-95 (2013).

4. Zhang, Y., Li, P., Feng, J. \& Wu, M. Dysfunction of NMDA receptors in Alzheimer's disease. Neurol. Sci. 37, 1039-1047 (2016).

5. Havekes, R. \& Abel, T. Genetic dissection of neural circuits and behavior in Mus musculus. Adv. Genet 65, 1-38 (2009).

6. Knott, E. P., Assi, M., Rao, S. N., Ghosh, M. \& Pearse, D. D. Phosphodiesterase inhibitors as a therapeutic approach to neuroprotection and repair. Int J. Mol. Sci. 18, 4 (2017)

7. Vandenberg, R. J. \& Aubrey, K. R. Glycine transport inhibitors as potential antipsychotic drugs. Expert Opin. Ther. Targets 5, 507-518 (2001).

8. Johnson, J. W. \& Ascher, P. Glycine potentiates the NMDA response in cultured mouse brain neurons. Nature 325, 529-531 (1987).

9. Mothet, J. P. et al. D-serine is an endogenous ligand for the glycine site of the N-methyl-D-aspartate receptor. Proc. Natl Acad. Sci. USA 97, 4926-4931 (2000)

10. Cubelos, B., Giménez, C. \& Zafra, F. Localization of the GLYT1 Glycine transporter at glutamatergic synapses in the rat brain. Cereb. Cortex. 15, 448-459 (2005).

11. Javitt, D. C. Glycine transport inhibitors in the treatment of schizophrenia. Handb. Exp. Pharm. 213, 367-399 (2012).

12. Kinney, G. G. et al. The glycine transporter type 1 inhibitor N-[3-(4'-fluorophenyl)-3-(4'-phenylphenoxy)propyl]sarcosine potentiates NMDA receptormediated responses in vivo and produces an antipsychotic profile in rodent behavior. J. Neurosci. 23, 7586-7591 (2003).

13. Le Pen, G. et al. Prepulse inhibition deficits of the startle reflex in neonata ventral hippocampal-lesioned rats: reversal by glycine and a glycine transporter inhibitor. Biol. Psychiatry 54, 1162-1170 (2003).

14. Martina, M. et al. Glycine transporter type 1 blockade changes NMDA receptor-mediated responses and LTP in hippocampal CA1 pyramidal cells by altering extracellular glycine levels. J. Physiol. 557, 489-500 (2004).

15. Papouin, T. et al. Synaptic and extrasynaptic NMDA receptors. Cell $\mathbf{1 5 0}$ 633-646 (2012)

16. Rosenberg, D. et al. Neuronal D-serine and glycine release via the Asc-1 transporter regulates NMDA receptor-dependent synaptic activity. J. Neurosci. 33, 3533-3544 (2013).

17. Li, Y. et al. Identity of endogenous NMDAR glycine site agonist in amygdala is determined by synaptic activity level. Nat. Commun. 4, 1760 (2013).

18. Curcio, L. et al. Reduced D-serine levels in the nucleus accumbens of cocainetreated rats hinder the induction of NMDA receptor dependent synaptic plasticity. Brain 136, 1216-1230 (2013).

19. Stevens, E. R. et al. D-serine and serine racemase are present in the vertebrate retina and contribute to the physiological activation of NMDA receptors. Proc. Natl Acad. Sci. USA 100, 6789-6794 (2003).

20. Panatier, A. et al. Glia-derived D-serine controls NMDA receptor activity and synaptic memory. Cell 125, 775-784 (2006).
21. Billard, J. M. Changes in serine racemase-dependent modulation of NMDA receptor: impact on physiological and pathological brain aging. Front Mol. Biosci. 5, 106 (2018).

22. Barnes, C. A. Long-term potentiation and the ageing brain. Philos. Trans. R. Soc Lond. B Biol. Sci. 358, 765-772 (2003).

23. Lynch, M. A. Age-related neuroinflammatory changes negatively impact on neuronal function. Front Aging Neurosci. 1, 6 (2010).

24. Jensen, O. \& Lisman, J. E. Hippocampal sequence-encoding driven by a cortical multi-item working memory buffer. Trends Neurosci. 28, 67-72 (2005).

25. Cavanagh, J. F. \& Frank, M. J. Frontal theta as a mechanism for cognitive control. Trends Cogn. Sci. 18, 414-421 (2014).

26. Fries, P., Reynolds, J. H., Rorie, A. E. \& Desimone, R. Modulation of oscillatory neuronal synchronization by selective visual attention. Science 291, 1560-1563 (2001).

27. Yamamoto, J, Suh, J., Takeuchi, D. \& Tonegawa, S. Successful execution of working memory linked to synchronized high-frequency gamma oscillations. Cell 157, 845-857 (2014)

28. Neuenschwander, S., Castelo-Branco, M., Baron, J. \& Singer, W. Feedforward synchronization: propagation of temporal patterns along the retinothalamocortical pathway. Philos. Trans. R. Soc. Lond. B Biol. Sci. 357, 1869-1876 (2002).

29. Ribry, U. Dynamics of thalamo-cortical network oscillations and human perception. Prog. Brain Res. 150, 127-142 (2005).

30. Buzsáki, G. et al. Hippocampal network patterns of activity in the mouse. Neuroscience 116, 201-211 (2003).

31. Bibbig, A. Traub, R. D. \& Whittington, M. A. Long-range synchronization of gamma and beta oscillations and the plasticity of excitatory and inhibitory synapses: a network model. J. Neurophysiol. 88, 1634-1654 (2002).

32. Klausberger, T. et al. Brain-state- and cell-type-specific firing of hippocampal interneurons in vivo. Nature 421, 844-848 (2003).

33. Olufsen, M. S., Whittington, M.A., Camperi, M. \& Kopell, N. New roles for the gamma rhythm: population tuning and preprocessing for the Beta rhythm. J. Comput Neurosci. 14, 33-54 (2003).

34. Bauer, E., Schafe, G. \& LeDoux, J. E. NMDA receptors and L-type voltage-gated calcium channels contribute to long-term potentiation and different components of fear memory formation in the lateral amygdala. J. Neurosci. 22, 5239-5249 (2002).

35. Morgan, S. L. \& Teyler, T. J. Electrical stimuli patterned after the theta-rhythm induce multiple forms of LTP. J. Neurophysiol. 86, 1289-1296 (2001).

36. Bollen, E. et al. Object memory enhancement by combining sub-efficacious doses of specific phosphodiesterase inhibitors. Neuropharmacology $\mathbf{9 5}$ 361-366 (2016).

37. Johansson, E. M., Reyes-lrisarri, E. \& Mengod, G. Comparison of CAMP-specific phosphodiesterase mRNAs distribution in mouse and rat brain. Neurosci. Lett. 525, 1-6 (2012).

38. Bolger, G. B. The PDE4 CAMP-specific phosphodiesterases: targets for drugs with antidepressant and memory-enhancing action. Adv. Neurobiol. 7, 63-102 (2017).

39. Wiescholleck, V. \& Manahan-Vaughan, D. PDE4 inhibition enhances hippocampal synaptic plasticity in vivo and rescues MK801-induced impairment of long-term potentiation and object recognition memory in an animal model of psychosis. Transl. Psychiatr. 2, e89 (2012).

40. Ahnaou, A. Huysmans, H. Jacobs, T. \& Drinkenburg, W. H. Cortical EEG oscillations and network connectivity as efficacy indices for assessing drugs with cognition enhancing potential. Neuropharmacology 86, 362-377 (2014).

41. Ahnaou, A., Huysmans, H., Biermans, R., Manyakov, N. V. \& Drinkenburg, W. H. I. M. Ketamine: differential neurophysiological dynamics in functional networks in the rat brain. Transl. Psychiatry 7, e1237 (2017).

42. Colgin, L. L. Do slow and fast gamma rhythms correspond to distinct functional states in the hippocampal network? Brain Res. 1621, 309-315 (2015).

43. Trimper, J. B., Galloway, C. R., Jones, A. C., Mandi, K. \& Manns, J. R. Gamma oscillations in rat hippocampal subregions dentate gyrus, CA3, CA1, and subiculum underlie associative memory encoding. Cell Rep. 21, 2419-2432 (2017).

44. Buzsáki, G. Large-scale recording of neuronal ensembles. Nat. Neurosci. $\mathbf{7}$ 446-451 (2004)

45. Colgin, L. L. Rhythms of the hippocampal network. Nat. Rev. Neurosci. 17 239-249 (2016)

46. Buzsáki, G. Theta oscillations in the hippocampus. Neuron 33, 325-340 (2002).

47. Robinson, J. et al. Optogenetic activation of septal glutamatergic neurons drive hippocampal theta rhythm. J. Neurosci. 36, 3016-3023 (2016). 
48. O'Neill, P. K., Gordon, J. A. \& Sigurdsson, T. Theta oscillations in the medial prefrontal cortex are modulated by spatial working memory and synchronize with the hippocampus through its ventral subregion. J. Neurosci. 33, 14211-14224 (2013)

49. Montgomery, S. M. \& Buzsáki, G. Gamma oscillations dynamically couple hippocampal CA3 and CA1 regions during memory task performance. Proc. Natl Acad. Sci. USA 104, 14495-14500 (2007).

50. Schomburg, E. W. et al. Theta phase segregation of input-specific gamma patterns in entorhinal-hippocampal networks. Neuron 84, 470-485 (2014).

51. Brun, V. H. et al. Place cells and place recognition maintained by direct entorhinal-hippocampal circuitry. Science 296, 2243-2246 (2002)

52. Steffenach, H. A., Sloviter, R. S., Moser, E. \& Moser, M. B. Impaired retention of spatial memory after transection of longitudinally oriented axons of HIPPOcampal CA3 pyramidal cells. Proc. Natl Acad. Sci. USA 99, 3194-3198 (2002).

53. Zheng, C., Bieri, K. W., Hwaun, E. \& Colgin, L. L. Fast gamma rhythms in the hippocampus promote encoding of novel object-place pairings. eNeuro. 3, ENEURO.0001-16.2016 (2016).

54. Nyhus, E. \& Curran, T. Functional role of gamma and theta oscillations in episodic memory. Neurosci. Biobehav Rev. 34, 1023-1035 (2010).

55. Després, O., Lithfous, S., Tromp, D., Pebayle, T. \& Dufour, A. Gamma oscillatory activity is impaired in episodic memory encoding with age. Neurobiol. Aging 52, 53-65 (2017).

56. Mably, A. J. \& Colgin, L. L. Gamma oscillations in cognitive disorders. Curr. Opin. Neurobiol. 52, 182-187 (2018).

57. Marshall, L., Helgadottir, H., Mölle \& Born, J. Boosting slow oscillations during sleep potentiates memory. Nature 444, 610-613 (2006).

58. Jacobson, T. K. et al. Hippocampal theta, gamma, and theta-gamma coupling: effects of aging, environmental change, and cholinergic activation. J. Neurophysiol. 109, 1852-1865 (2013).

59. McNaughton, N., Ruan, M. \& Woodnorth, M. A. Restoring theta-like rhythmicity in rats restores initial learning in the Morris water maze. Hippocampus 16, 1102-1110 (2006).

60. Reinhart, R. M. G. Disruption and rescue of interareal theta phase coupling and adaptive behavior. Proc. Natl Acad. Sci. USA 114, 11542-1154 (2017).

61. Javitt, D. C. et al. Adjunctive high-dose glycine in the treatment of schizophrenia. Int J. Neuropsychopharmacol. 4, 385-391 (2001).

62. Paoletti, P., Bellone, C. \& Zhou, Q. NMDA receptor subunit diversity: impact on receptor properties, synaptic plasticity and disease. Nat. Rev. Neurosci. 14, 383-400 (2013).

63. Singer, P., Feldon, J. \& Yee, B. K. The glycine transporter 1 inhibitor SSR504734 enhances working memory performance in a continuous delayed alternation task in C57BL/6 mice. Psychopharmacology 202, 371-384 (2009).

64. Turpin, F., Dallérac, G. \& Mothet, J. P. Electrophysiological analysis of the modulation of NMDA-receptors function by D-serine and glycine in the central nervous system. Methods Mol. Biol. 794, 299-312 (2012).

65. Housaly, M. D. Underpinning compartmentalised CAMP signalling through targeted CAMP breakdown. Trends Biochem Sci. 35, 91-100 (2010).

66. Kawazaki, H. \& Takasaki, K. Electroencephalographic study with rolipram (ME 3167), a selective inhibitor of adenosine 3',5'-monophosphate Phosphodiesterase, in rabbits. Nihon Yakurigaku Zasshi 97, 221-229 (1991).

67. Pape, H. C., Narayanan, R. T., Smid, J., Stork, O. \& Seidenbecher, T. Theta activity in neurons and networks of the amygdala related to long-term fear memory. Hippocampus 15, 874-880 (2005).

68. Bliss, T. V. \& Collingridge, G. L. A synaptic model of memory: long-term potentiation in the hippocampus. Nature 361, 31-39 (1993).

69. Collingridge, G. L. et al. The NMDA receptor as a target for cognitive enhancement. Neuropharmacology 64, 13-26 (2013).

70. Chen, L., Muhlhauser, M. \& Yang, C. R. Glycine tranporter-1 blockade potentiates NMDA-mediated responses in rat prefrontal cortical neurons in vitro and in vivo. J. Neurophysiol. 89, 691-703 (2003).

71. Depoortère, R. et al. Neurochemical, electrophysiological and pharmacological profiles of the selective inhibitor of the glycine transporter-1 SSR504734, a potential new type of antipsychotic. Neuropsychopharmacology $\mathbf{3 0}$, 1963-1985 (2005)

72. Leonetti, M. et al. Steinberg R. 2-chloro-N-[(S)-phenyl [(2S)-piperidin-2-yl] methyl]-3-trifluoromethyl benzamide, monohydrochloride, an inhibitor of the glycine transporter type 1, increases evoked-dopamine release in the rat nucleus accumbens in vivo via an enhanced glutamatergic neurotransmission. Neuroscience 137, 555-564 (2006).

73. Nishikawa, H., Inoue, T., Izumi, T., Nakagawa, S. \& Koyama, T. SSR504734, a glycine transporter-1 inhibitor, attenuates acquisition and expression of contextual conditioned fear in rats. Behav. Pharmacol. 21, 5-6 (2010).

74. Singer, P., Zhang, W. \& Yee, B. K. SSR504734 enhances basal expression of prepulse inhibition but exacerbates the disruption of prepulse inhibition by apomorphine. Psychopharmacology 230, 309-317 (2013).

75. Zhang, Z., Gong, N., Wang, W., Xu, L. \& Xu, T. L. Bell-shaped D-serine actions on hippocampal long-term depression and spatial memory retrieval. Cereb. Cortex 18, 2391-2401 (2008)

76. Mothet, J. P. et al. A critical role for the glial-derived neuromodulator D-serine in the age-related deficits of cellular mechanisms of learning and memory. Aging Cell 5, 267-274 (2006).

77. Junjaud, G., Turpin, F., Mothet, J. P. \& Billard, J. M. Age-related effects of the neuromodulator D-serine on neurotransmission and synaptic potentiation in the CA1 hippocampal area of the ra'. J. Neurochem. 98, 1159-1166 (2006).

78. Duffy., S., Labrie, V. \& Roder, J. C. D-serine augments NMDA-NR2B receptordependent hippocampal long-term depression and spatial reversal learning. Neuropsychopharmacology 33, 1004-1018 (2008).

79. Han, H., Peng, Y. \& Dong, Z. D-Serine rescues the deficits of hippocampal longterm potentiation and learning and memory induced by sodium fluoroacetate'. Pharm. Biochem Behav. 133, 51-56 (2015).

80. Burke, S. N. \& Barnes, C. A. Neural Plasticity in the Ageing Brain. Nat. Rev. Neurosci. 7, 30-40 (2006).

81. Segovia, G., Porras, A., Del Arco, A. \& Mora, F. Glutamatergic Neurotransmission in Aging: A Critical. Perspective. Mech. Ageing Dev. 122, 1-29 (2001).

82. Andersen, J. D. \& Pouzet, B. Spatial memory deficits induced by perinatal treatment of rats with PCP and reversal effect of D-serine. Neuropsychopharmacology 29, 1080-1090 (2004).

83. Zhu, G., Liu, Y., Wang, Y., Bi, X. \& Baudry, M. Different patterns of electrical activity lead to long-term term potentiation by activating different intracellular pathways. J. Neurosci. 35, 621-633 (2015).

84. Nong, Y. et al. 'Glycine binding primes NMDA receptor internalization. Nature 422, 302-307 (2003).

85. Lo, E. H. et al. Alterations in $\mathrm{K}+$ evoked profiles of neurotransmitter and neuromodulator amino acids after focal ischemia-reperfusion. Neuroscience $\mathbf{8 3}$, 449-458 (1998)

86. $\mathrm{Wu}, \mathrm{S}$. Z. et al. Induction of serine racemase expression and D-serine release from microglia by amyloid $\beta$-peptide. J. Neuroinflammation 1, 2 (2004).

87. Buchanan, R. W. et al. The cognitive and negative symptoms in schizophrenia trial (CONSIST): The efficacy of glutamatergic agents for negative symptoms and cognitive impairments. Am. J. Psychiatry 164, 1593-1602 (2007).

88. Barad, M., Bourtchouladze, R., Winder, D. G., Golan, H. \& Kandel, E. Rolipram, a type IV-specific phosphodiesterase inhibitor, facilitates the establishment of long-lasting long-term potentiation and improves memory. Proc. Natl Acad. Sci. USA 95, 15020-15025 (1998).

89. Vecsey, C. G. et al. Sleep Deprivation Impairs CAMP Signalling in the Hippocampus. Nature 461, 1122-1125 (2009).

90. Wimmer, M. E., Blackwell, J. M. \& Abel, T. Rolipram Treatment During Consolidation Ameliorates Long-Term Object Location Memory in Aged Male Mice. Neurobiol. Learn Mem. 169, 107168 (2020).

91. Gong, B. et al. Persistent improvement in synaptic and cognitive functions in an Alzheimer mouse model after rolipram treatment. J. Clin. Invest 114, 1624-1634 (2004)

92. Brown, G. P. et al. Long-term potentiation induced by theta frequency stimulation is regulated by a protein phosphatase-1-operated gate. J. Neurosci. 20, 7880-7887 (2000)

93. Vitolo, O. V. et al. Amyloid beta peptide inhibition of the PKAVCREB pathway and long-term potentiation: reversibility by drugs that enhance CAMP signaling. Proc. Natl Acad. Sci. USA 99, 13217-13221 (2002).

94. Huang, Y. Y. \& Kandel, E. R. Recruitment of long-lasting and protein kinase Adependent long-term potentiation in the CA1 region of hippocampus requires repeated tetanization. Learn Mem. 1, 74-82 (1994).

95. Park, P. et al. Calcium-permeable AMPA receptors mediate the induction of the protein kinase a-dependent component of long-term potentiation in the hippocampus. J. Neurosci. 36, 622-631 (2016). 